\title{
LAQUEADURA INTRAPARTO E DE INTERVALO
}

Arlete Maria dos Santos Fernandes*, Aloísio José Bedone, larissa Capochin Paes Leme, Elza Mitiko Yamada

Trabalho realizado na Área de Tocoginecologia, Faculdade de Ciências Médicas, Pontifícia Universidade Católica de Campinas

\begin{abstract}
RESUMO
O Brasil é um dos países com alta prevalência de laqueadura tubária, sendo freqüente sua realização durante o parto. Nos últimos anos, tem-se notado aumento da busca por reversão, principalmente entre mulheres jovens.

OBjetivos. Estudar, em amostra de mulheres laqueadas, as características relacionadas ao procedimento, determinar a frequeência de execução intraparto, medir as taxas de satisfação e arrependimento com o método.

Métodos. Foram entrevistadas 335 mulheres laqueadas. As variáveis estudadas foram as relacionadas ao procedimento: idade na laqueadura, se intraparto (vaginal ou cesárea) ou de intervalo (fora do período de parto e puerpério), serviço no qual foi realizada, pagamento pelo procedimento, motivo declarado para a opção pelo método, e as relacionadas à satisfação/ arrependimento: desejo de gravidez após esterilização, procura por tratamento e realização da cirurgia de reversão. As mulheres foram divididas em dois grupos, laqueadas intraparto e intervalo, avaliando-se associação das variáveis pelo teste Exato de Fisher e Qui-quadrado com correçã̃o de Yates. O estudo obteve aprovação do Comitê de Ética.

Resultados. Predominaram mulheres brancas, com mais de 35 anos, unidas, com baixa escolaridade e 43,5\% esterilizadas antes dos 30 anos. Haviam realizado laqueadura intraparto 245 mulheres, 91,2\% daquelas com parto cesárea e 44,6\% com parto vaginal. Nos dois grupos, laqueadas intraparto e de intervalo, estavam satisfeitas com o método $82 \%$ e $80,8 \%$ das mulheres respectivamente. Apesar de $14,6 \%$ terem referido desejo de engravidar alguma vez após esterilização, consultaram por esterilidade 3,4\% e 2,3\%, respectivamente, e uma mulher submeteu-se à cirurgia de reversão. Os motivos mais freqüentes para a escolha do método foram a satisfação com a prole (35,5\% e 46,7\%) e a indicação médica (41,6\% e 32,2\%), respectivamente.
\end{abstract}

Conclusão. A laqueadura intraparto foi maior quando o último parto foi cesárea. Não se detectou diferença nas taxas de satisfação e arrependimento após o procedimento entre os grupos.

UnITERMOS: Anticoncepção. Laqueadura tubária. Parto cesárea.

\section{INTRODUÇÃO}

A laqueadura tem sido o método de contracepção eleito por muitos casais em todo o mundo pela eficácia e segurança. Nos Estados Unidos, é estimado que um milhão de procedimentos de laqueadura tubária sejam realizados anualmente, sendo que metade deles são realizados logo após o parto'. Se, por um lado, existem relatos de altas taxas de satisfação com método definitivo ${ }^{2,3}$, por outro, as taxas de arrependimento em estudos prospectivos giram em torno de $3 \%$ nos primeiros dois anos de seguimento ${ }^{4}$. No Brasil, enquanto os dados de prevalência mostram que cerca de $40 \%$ das mulheres unidas estão laqueadas ${ }^{5}$, tem sido observado aumento no número de mulheres arrependidas que buscam tratamento nos serviços de esterilidade ${ }^{6}$. Essa situação tem preocupado os serviços de saúde, já que nem sempre o tratamento de reversão é possível e, mesmo quando é realizada a reanastomose tubária, as taxas de gravidez são limitadas?. Àquelas mulheres que não engravidam, resta a fertilização in vitro com transferência de embriões (FIV-TE), tratamento de alto custo ao qual pequeno número de mulheres têm acesso.

Em meio a isso, as taxas nacionais de partos cesárea têm sido progressivamente altas ${ }^{8}$. Devido ao grande número de laqueaduras realizadas durante a internação para assistência ao parto, principalmente durante cesárea², em 1996, foi aprovada a Lei no 9263 que passou a garantir acesso aos métodos de esterilização por meio dos serviços públicos ${ }^{9}$. A referida lei buscou promover a dissociação entre o procedimento e o parto, permitindo a realização da esterilização a homens e mulheres maiores de 25 anos ou pelo menos com dois filhos vivos. A lei proíbe o procedimento durante a assistência ao parto e aborto, postergando-o por período mínimo de 60 dias a partir da opção pelo método, devendo ser realizada, nesse intervalo, a orientação para os métodos de contracepção reversíveis. A única exceção que possibilita a sua realização intraparto é a indicação por cesáreas sucessivas?.

Os objetivos deste estudo foram avaliar as características do procedimento de laqueadura em amostra de mulheres esterilizadas, determinar a freqüência daqueles realizados intraparto, vaginal ou cesárea, avaliar o motivo alegado para a opção e medir a freqüência de mulheres satisfeitas e arrependidas com o método definitivo nos grupos de laqueadura intraparto e de intervalo.

\section{Métodos}

O estudo foi de coorte transversal. No período de fevereiro a outubro de 200I, foram recrutadas mulheres que preencheram os critérios de inclusão e aceitaram participar do estudo entre as que 
Tabela I - Características relacionadas ao procedimento nos grupos de mulheres com laqueadura intraparto e de intervalo

\begin{tabular}{|c|c|c|c|c|c|}
\hline \multirow{3}{*}{$\begin{array}{l}\text { Características das mulheres } \\
\text { Idade da laqueadura } \\
\text { Até } 29 \text { anos } \\
\quad \geq 30 \text { anos }\end{array}$} & \multicolumn{4}{|c|}{ Momento da laqueadura } & \multirow[b]{3}{*}{ NS } \\
\hline & \multicolumn{2}{|c|}{ Intraparto } & \multicolumn{2}{|c|}{ Intervalo } & \\
\hline & $\begin{array}{l}104 \\
14 \mid\end{array}$ & $\begin{array}{l}42,4 \\
57,6\end{array}$ & $\begin{array}{l}42 \\
48\end{array}$ & $\begin{array}{l}46,7 \\
53,3\end{array}$ & \\
\hline \multicolumn{6}{|l|}{ Internação } \\
\hline $\begin{array}{l}\text { SUS } \\
\text { Convênio/particular } \\
\text { H. universitário }\end{array}$ & $\begin{array}{l}122 \\
97 \\
26\end{array}$ & $\begin{array}{l}49,8 \\
39,6 \\
10,6\end{array}$ & $\begin{array}{l}56 \\
20 \\
14\end{array}$ & $\begin{array}{l}62,2 \\
22,2 \\
15,6\end{array}$ & 0,009 \\
\hline \multicolumn{6}{|l|}{ Pagou pela laqueadura } \\
\hline $\begin{array}{l}\text { Nấo } \\
\text { Sim }\end{array}$ & $\begin{array}{l}128 \\
117\end{array}$ & $\begin{array}{l}52,2 \\
47,8\end{array}$ & $\begin{array}{l}65 \\
25\end{array}$ & $\begin{array}{l}72,2 \\
27,8\end{array}$ & 0,001 \\
\hline \multicolumn{6}{|l|}{ Satisfeita com a laqueadura } \\
\hline $\begin{array}{l}\text { Não } \\
\text { Sim }\end{array}$ & $\begin{array}{l}48 \\
197\end{array}$ & $\begin{array}{l}19,6 \\
80,4\end{array}$ & $\begin{array}{l}14 \\
76\end{array}$ & $\begin{array}{l}15,6 \\
84,4\end{array}$ & NS \\
\hline $\begin{array}{l}\text { Pensou em gestar após laquea } \\
\text { Não } \\
\text { Sim }\end{array}$ & $\begin{array}{l}208 \\
37\end{array}$ & $\begin{array}{l}84,5 \\
15,5\end{array}$ & $\begin{array}{l}79 \\
11\end{array}$ & $\begin{array}{l}87,8 \\
12,2\end{array}$ & NS \\
\hline \multicolumn{6}{|l|}{$\begin{array}{l}\text { Consultoupor } \\
\text { esterilidade }\end{array}$} \\
\hline $\begin{array}{l}\text { Não } \\
\text { Sim }\end{array}$ & $\begin{array}{c}28 \\
9\end{array}$ & $\begin{array}{l}75,7 \\
24,3\end{array}$ & $\begin{array}{l}10 \\
1\end{array}$ & $\begin{array}{c}90,9 \\
9,1\end{array}$ & NS \\
\hline \multicolumn{6}{|l|}{ Fez reversão da laqueadura } \\
\hline $\begin{array}{l}\text { Não } \\
\text { Sim }\end{array}$ & $\begin{array}{c}36 \\
1\end{array}$ & $\begin{array}{l}97,3 \\
2,7\end{array}$ & $\begin{array}{c}11 \\
0\end{array}$ & $\begin{array}{c}100,0 \\
-\end{array}$ & NS \\
\hline N & & & & & 335 \\
\hline
\end{tabular}

*Teste Exato de Fisher; NS: não significativo

aguardavam atendimento no ambulatório geral de Ginecologia. Foram incluídas mulheres laqueadas com 25 anos ou mais e antecedente de pelo menos dois partos anteriores, às quais foi aplicado questionário estruturado por entrevistadora treinada. Observou-se que o número de mulheres com laqueadura intraparto excedeu rapidamente as de intervalo, tornando desnecessário cálculo amostral para o grupo estudado. As variáveis estudadas foram as características relacionadas ao procedimento como idade na laqueadura, se intraparto (vaginal ou cesárea) ou se de intervalo (fora do período de parto e puerpério), serviço no qual foi realizado o procedimento, pagamento pelo procedimento, motivo declarado para a opção pelo método e as características relacionadas à satisfação/arrependimento com o método, se desejou engravidar após o procedimento, se consultou por esterilidade e se realizou a cirurgia de reversão.

Após o cálculo de freqüência para as características sociodemográficas e antecedente de uso de métodos anticoncepcionais reversíveis, as mulheres foram divididas em dois grupos segundo o momento de realização da laqueadura, intraparto e de intervalo. Para a avaliação da associação das variáveis estudadas em relação aos grupos, utilizou-se o teste Exato de Fisher ou, quando necessário, o teste Qui-quadrado com correção de Yates ${ }^{10}$. Foi considerado nível de significância de 5\%. O estudo teve a aprovação do Comitê de Ética em Pesquisa da FCM/PUC-Campinas.

\section{Resultados}

A análise inicial das 335 mulheres entrevistadas mostrou 45,6\% com até 44 anos. A maior parte delas era branca e 87,5\% tinha até 8 anos de escolaridade. A proporção de mulheres unidas foi $86 \%$ e aproximadamente um terço delas referiu antecedente de casamento anterior.

Mais da metade de mulheres tinha dois a cinco filhos, um quarto era de grandes multíparas (seis filhos ou mais) e 17,6\% tinham um ou nenhum filho vivo. $O$ antecedente de pelo menos uma cesárea anterior ocorreu em $64,2 \%$ da amostra. Por outro lado, o último parto havia sido por meio de cesárea em 61,2\% das mulheres.

Quando perguntadas sobre o antecedente de uso dos métodos reversíveis modernos de anticoncepção, 66,9\% delas já haviam utilizado algum deles. Quanto ao método utilizado, o anticoncepcional oral combinado (ACO) foi o referido por $85,7 \%$, seguido do condom e do DIU por cerca de $15 \%$, enquanto todos os demais métodos foram citados por menos de 10\% das mulheres. Um terço do total de mulheres nunca havia utilizado qualquer método reversível anteriormente à laqueadura tubária.

A Tabela I mostra os resultados de análise das mulheres distribuídas em dois grupos: 245 (73,1\%) com laqueadura intraparto e 90 mulheres $(26,9 \%)$ com laqueadura de intervalo. 
Tabela 2 - Distribuição das mulheres que realizaram o procedimento intraparto e no intervalo segundo o motivo declarado para a realização da laqueadura

\begin{tabular}{|c|c|c|c|}
\hline \multirow[t]{2}{*}{ Motivo da laqueadura } & \multicolumn{2}{|c|}{ Laqueadura* } & \multirow[t]{2}{*}{ Total (\%) } \\
\hline & $\begin{array}{c}\text { Intraparto } \\
\mathrm{n}(\%)\end{array}$ & $\begin{array}{c}\text { Intervalo } \\
n(\%)\end{array}$ & \\
\hline Satisfeita c/ prole & $87(35,5)$ & $\overline{42(46,7)}$ & $129(38,5)$ \\
\hline Doença & $52(21,2)$ & $16(17,8)$ & $68(20,3)$ \\
\hline Médico indicou & $4 \mid(16,7)$ & $13(14,4)$ & $54(16,1)$ \\
\hline Condição financeira & $21(8,6)$ & $6(6,7)$ & $27(8,0)$ \\
\hline Intolerância a MAC & $10(4,1)$ & $5(5,6)$ & $15(4,5)$ \\
\hline Marido & $10(4,1)$ & $2(2,2)$ & $12(3,6)$ \\
\hline Idade & $9(3,7)$ & $2(2,2)$ & ||$(3,3)$ \\
\hline Cesáreas repetidas & $9(3,7)$ & - & $9(2,7)$ \\
\hline Partos difíceis & $6(2,4)$ & $2(2,2)$ & $8(2,4)$ \\
\hline Aproveitou outra cirurgia & - & $2(2,2)$ & $2(0,6)$ \\
\hline $\mathbf{N}$ & $245(73, I)$ & $90(26,9)$ & $335(100,0)$ \\
\hline
\end{tabular}

*Qui-quadrado com correção de Yates: p=0,5।

Tabela 3 - Distribuição das mulheres laqueadas intraparto segundo tipo de último parto e o motivo declarado para a realização da laqueadura

\begin{tabular}{|c|c|c|c|}
\hline \multirow[t]{2}{*}{ Motivo da laqueadura } & \multicolumn{2}{|c|}{ Último parto* } & \multirow[t]{2}{*}{ Total (\%) } \\
\hline & $\begin{array}{c}\text { Cesárea } \\
\text { n(\%) }\end{array}$ & $\begin{array}{c}\text { Vaginal } \\
\mathrm{n}(\%)\end{array}$ & \\
\hline Satisfeita c/ prole & $62(33,2)$ & $\overline{25(43, I)}$ & $87(35,5)$ \\
\hline Doença & $38(20,3)$ & $14(24,1)$ & $52(21,2)$ \\
\hline Médico indicou & $34(18,2)$ & $7(12,1)$ & $4 \mid(16,7)$ \\
\hline Condição financeira & $16(8,6)$ & $5(8,6)$ & $21(8,6)$ \\
\hline Marido & $9(4,8)$ & I $(1,7)$ & $10(4,1)$ \\
\hline Intolerância a MAC & $9(4,8)$ & I $(1,7)$ & $10(4,1)$ \\
\hline Cesáreas repetidas & $9(4,8)$ & - & $9(3,7)$ \\
\hline Idade & $7(3,7)$ & $2(3,5)$ & $9(3,7)$ \\
\hline Partos difíceis & $3(1,6)$ & $3(5,2)$ & $6(2,4)$ \\
\hline Total & $187(76,3)$ & $58(23,6)$ & $245(100,0)$ \\
\hline
\end{tabular}

*Qui-quadrado com correção de Yates: $p=0,63$

Com relação à idade, $42,4 \%$ do grupo de laqueadas intraparto e $46,7 \%$ do de intervalo tinham menos de 30 anos no momento da realização da laqueadura. Não houve diferenças entre os grupos com relação à idade das mulheres no momento da laqueadura.

A forma de internação/cobertura do procedimento foi diferente quando se comparou os dois grupos $(p<0,01)$. Quando o procedimento foi realizado pelo SUS, mais mulheres tiveram laqueadura de intervalo; ao contrário, quando o procedimento foi realizado por internação via convênio/particular, houve maior proporção de mulheres com laqueadura intraparto. Nos hospitais universitários, os procedimentos intraparto e de intervalo foram realizados em 10\% e 15\% das mulheres, respectivamente. Houve maior número de mulheres que referiram pagamento pela laqueadura "por fora" quando o procedimento foi realizado intraparto em relação ao de intervalo $(p<0,00$ I) (Tabela I).

A maioria das mulheres, $80,4 \%$ do grupo com laqueadura intraparto e $84,4 \%$ do grupo de laqueadura de intervalo, estava satisfeita com o método e as demais referiram-se não satisfeitas. Quando perguntadas se haviam desejado engravidar alguma vez após a laqueadura, 15,5\% e 12,2\%, respectivamente, referiram que sim. Apesar disso, dez mulheres consultaram por esterilidade, nove do grupo de laqueadas intraparto e uma do grupo de intervalo. Somente uma mulher do grupo de laqueadas intraparto submeteu-se à cirurgia de reversão. Não houve diferença nas taxas de satisfação e arrependimento entre os dois grupos (Tabela I).

Os três motivos mais citados para haver optado pela laqueadura foram estar satisfeita com a prole $(38,5 \%)$, por doença concomitante $(20,3 \%)$ e quando o médico indicou $(16,1 \%)$. Todos os demais motivos foram citados por menos de $10 \%$ das mulheres. Não houve diferença nos motivos alegados para a opção pelo método definitivo entre as mulheres que laquearam intraparto ou no intervalo (Tabela 2).

A Tabela 3 mostra a distribuição das mulheres laqueadas intraparto, segundo o motivo alegado para a realização do procedimento. Das 245 mulheres com laqueadura intraparto, 187 (76,3\%) tiveram parto cesárea e 58 (23,6\%) parto vaginal. A distribuição das mulheres segundo tipo de último parto e motivo alegado para ter optado pela laqueadura não mostrou diferenças entre os grupos. 
Fernandes AMS et AL.

\section{Discussão}

A amostra estudada foi predominantemente de mulheres brancas, com baixa escolaridade e unidas em primeiro relacionamento. Caracterizou-se por ter semelhante proporçãa de mulheres com idade superior e inferior a 45 anos, tornando possível avaliar os procedimentos de esterilização realizados no período entre as décadas imediatamente anteriores e os primeiros anos da existência da lei de esterilização. Quase metade das mulheres havia sido laqueada antes dos 30 anos de idade, confirmando prevalência de mulheres jovens esterilizadas compatível com o descrito em pesquisa demográfica nacional $\left.\right|^{5}$. A análise mostrou que, em contrapartida, um terço delas nunca havia utilizado qualquer método anticoncepcional reversível, proporção similar à registrada em pesquisa demográfica ${ }^{5}$.

Dos procedimentos realizados intraparto, $76 \%$ o foram durante parto cesárea. Este fato reproduz a importante vinculação da realização da laqueadura ao momento do parto no Brasil, onde o setor público tem sido utilizado em grande parte das internações e, ainda assim, existe pagamento pelo procedimento; nesta amostra $65,5 \%$ e $42 \%$ das internações, respectivamente. Estudo nacional de base populacional já havia detectado que $74 \%$ das esterilizações haviam sido realizadas intraparto, destas, $80 \%$ durante o parto cesárea, sendo maior quanto maior a escolaridade da mulher, chegando a $82 \%$ entre mulheres com 12 anos ou mais de estudo ${ }^{2}$. Nesta amostra, com $87,5 \%$ das mulheres com até oito anos de estudo, não foi possível qualquer avaliação em relação à característica de escolaridade.

Até o momento da entrada em vigor da lei de esterilização, não existia qualquer cobertura do setor público ou de convênios para sua realização, o que explica que a laqueadura manteve-se à margem dos procedimentos médicos oficiais. Este fato originou o comportamento, por parte dos médicos, de realizar a laqueadura aproveitando a cesárea e com cobrança "por fora". Por outro lado, havia a demanda das mulheres, inclusive as menos favorecidas do ponto de vista socioeconômico, que viam na laqueadura a possibilidade de ascensão social e de melhoria das condições de vida ${ }^{3}$. Essas duas atitudes acabaram por "oficializar" a prática de se indicar cesárea para realizar a laqueadura.

A percentagem de mulheres insatisfeitas com o método foi de 18,5\%. Não houve diferença entre o grupo de mulheres que realizou a cirurgia durante ou fora do ciclo grávido-puerperal. Muito provavelmente, a maioria das mulheres laqueadas intraparto teve a indicação da cesárea para realizar a esterilização. Esse foi um resultado constatado nas 205 mulheres com último parto cesárea, das quais 187 foram esterilizadas intraparto. No grupo de mulheres insatisfeitas com a laqueadura, a maioria referiu haver desejado, em algum momento, engravidar novamente. Pelo pequeno número das que buscaram tratamento de esterilidade e o fato de uma chegar à reversão da laqueadura, é possível imaginar que, apesar de se arrependerem, nem sempre as mulheres buscam tratamento. Pode-se cogitar que o acesso possa ser difícil ou que as mulheres não tenham informação, as condições socioeconômicas podem ser limitantes, ou até, apesar de existir o desejo, ele não seja suficiente para mudar o projeto anterior, já que também é trabalhoso voltar a se preocupar com o controle reprodutivo. Também houve mulheres que, mesmo que não tivessem desejado nova gravidez, estavam insatisfeitas com o método definitivo. Apesar de eficaz anticoncepção e de ser capaz de retirar da mulher o ônus da preocupação com a reprodução, a laqueadura também deve ser associada a outros efeitos que podem trazer desconforto e, desse modo, as mulheres verbalizam a insatisfação.

O que leva os casais a optar pelo método definitivo em algum momento da vida? Apesar da amostra ser constituída por mulheres que freqüentam hospital público e com característica de baixa escolaridade, poucas referiram fator econômico para justificar sua opção. A satisfação com o número de filhos foi citada mais vezes e é compreensível pela maioria ter pelo menos dois filhos vivos e existir um quarto de mulheres com mais de seis filhos. Além disso, é uma justificativa genuína e deve ser respeitada. Entretanto, chamou a atenção o fato dos profissionais médicos contribuírem com tantas justificativas. $\mathrm{O}$ aconselhamento médico, se incluirmos também a justificativa "doença", soma 36\% das indicações. Quando o grupo de mulheres laqueadas intraparto foi avaliado, o motivo "cesáreas repetidas" somado às demais indicações pelo médico elevou para $43 \%$ as justificativas médicas para realização da laqueadura durante a cesárea. Nessa população, a indicação médica superou a satisfação com a prole como justificativa para a laqueadura. A opção pelo método anticonceptivo definitivo por indicação médica pode significar prática iatrogênica quando se verifica que mais de $40 \%$ das mulheres foram laqueadas antes dos 30 anos de idade.

Com relaçãao às taxas de satisfação e arrependimento, esperarse-ia que mulheres com partos vaginais, maior prole e idade avançada tivessem indicação mais adequada de laqueadura e, portanto, estivessem mais satisfeitas com a opção de esterilização quando comparadas com mulheres que, em razão de cesáreas sucessivas, acabam sendo esterilizadas mais precocemente. Esse fato não foi detectado neste estudo; a indicação de cesárea para realizar laqueadura aparentemente não aumentou o risco de insatisfação ou arrependimento. É provável que isto tenha ocorrido porque nesta amostra foram entrevistadas, relativamente, poucas mulheres com menos de 35 anos de idade (I2\%).

Se, por um lado, a lei foi um avanço importante para regulamentar o procedimento no Brasil, por outro, tornou proibitiva sua realização no momento do parto, penalizando mulheres que demandam concretizar o procedimento durante essa internação. Esta determinação pode produzir efeitos não desejados. Ao proibir a laqueadura pós-parto (aquela realizada por via infraumbilical nas primeiras horas após o parto), pode-se estar propiciando o aumento do número de cesáreas, durante as quais seriam realizadas laqueaduras sem anotação em prontuário. Note-se que a indicação de cesárea com o intuito de se realizar a laqueadura permanece sendo conduta médica condenável pela maior morbidade do ato cirúrgico.

\section{Conclusão}

Os resultados deste estudo mostraram que, mesmo analisando uma população de mulheres com maior idade e maior prole, a taxa de insatisfação situou-se em torno de $18 \%$. Se atentarmos apenas para o desejo de nova gravidez em mulheres esterilizadas, a taxa de $14 \%$ 
observada mostrou-se bastante elevada, principalmente pelos aspectos relativos à faixa etária das mulheres do estudo. Pode-se especular que essas taxas seriam mais elevadas se maior número de mulheres jovens fosse avaliado.

É possível que outros fatores, que não a realização da laqueadura por ocasião do parto, possam ser determinantes da taxa de arrependimento. Outros estudos devem ser realizados, com mulheres laqueadas sob vigência da nova lei para avaliar se o grau de arrependimento está relacionado com o momento da esterilização ou com outros fatores, entre eles, a idade da mulher, novo casamento e o óbito dos filhos. O que se pode inferir deste estudo é, tão somente, que a realização de laquedura por ocasião do parto não significa, necessariamente, aumento das taxas de arrependimento, pelo menos em populações com as características das mulheres desta amostra.

Conflito de interesse: não há

\section{SUMMARY}

\section{INTRAPARTUM AND INTERVAL TUBAL STERILIZATION: CHARACTERISTICS CORRELATED WITH THE PROCEDURE AND REGRET IN A SAMPLE OF WOMEN FROM A PUBLIC HOSPITAL}

BACKGROUND. Brazil is a country with a high prevalence oftubal ligation, which is frequently performed at the time of delivery. In recent years, an increase in tubal reversal has been noticed, primarily amongyoungwomen.

OBJECTNES. To study characteristics correlated with the procedure, determine frequency of intrapartum tubal ligation, measure patient satisfaction rates and tubal sterilization regret, in a sample of post-tubal patients.

Methods. Three hundred and thirty-five women underwent tubal ligation. The variables studied were related to the procedure: age at tubal ligation, whether ligation was performed intrapartum (vaginal or cesarean section) or after an interval (other than the intrapartum and puerperal period), health service performing the sterilization, medical expenses paid for the procedure, reason stated for choosing the method and causes related to satisfaction/regret: desire to become pregnant after sterilization, search for treatment and performance of tubal ligation reversal. The women were divided into two groups, a group undergoing ligation in the intrapartum period and a second group ligated after an interval, to evaluate the association between variables by using Fisher's exact test and chi-squared calculation with Yates' correction. The study was approved by the Ethics Committee of the institution.

RESULTS. There was a predominance of Caucasian women over 35 years of age, married, and with a low level of education of which $43.5 \%$ had undergone sterilization before 30 years of age. Two hundred and forty-five women underwent intrapartum tubal ligation, 91.2\% of them had cesarean delivery and $44.6 \%$ vaginal delivery. In both groups undergoing intrapartum tubal ligation and ligation after an interval, $82.0 \%$ and $80.8 \%$ reported satisfaction with the method. Although $14.6 \%$ expressed a desire to become pregnant at some time after sterilization, consultation regarding sterility occurred in $3.4 \%$ and $2.3 \%$, respectively, and one woman underwenttubal reversal. The mostfrequentreasonsforchoosing the method were satisfaction with the number of offspring (35.5\% and 46.7\%) and medical indication (41.6\% and 32.2\%), respectively.

CONCLUSION. The incidence of intrapartum tubal ligation was higher when the last delivery had been a cesarean section. No difference was detected among the groups regarding rates of satisfaction and regret after performance of the sterilization procedure. [Rev Assoc Med Bras 2006; 52(5): 323-7]

KEY wORDS: Contraception. Tubal ligation. Cesarean delivery.

\section{REFERÊNCIAS}

I. Mackay AP, Kieke BA Jr, Koonin LM, Beattie K. Tubal sterilization in the United States, 1994- 1996. Fam Plann Perspect. 200 I;33: I 61-5.

2. Minella LS. Aspectos positivos e negativos da esterilização tubária do ponto de vista de mulheres esterilizadas. Cad Saúde Publica. 1998; | 4(SI):69-79.

3. Osis MJD, Faúndes A, Sousa MH, Bailey P. Conseqüências do uso de métodos anticoncepcionais na vida das mulheres: o caso da laqueadura tubária. Cad Saúde Publica. 1999; 1 5:521-32.

4. Grubb GS, Peterson HB, Layde PM, Rubin GL. Regret after decision to have a tubal sterilization. Fertil Steril. 1985;44:248-53.

5. Pesquisa Nacional de Demografia e Saúde. Rio de Janeiro: BENFAM; 1996.

6. Petta CA, Dantas C, Hidalgo MM, Bahamondes L. Solicitações de reversão da laqueadura em um serviço de Esterilidade: o problema continua. Reprod Clim. 2000; 1 5:214-7.

7. Fernandes AMS, Arruda MS, Palhares MAR, Benetti-Junior ND, Moreira CM. Seguimento de mulheres laqueadas arrependidas em serviço público de esterilidade conjugal. Rev Bras Ginecol Obstet. 200 I;23:69-73.

8. Barros FC, Vaughan JP, Victora CG, Huttly SRA. Epidemic of cesarean sections in Brazil. Lancet. 1991;338:167-69.

9. Brasil. Lei ordinária n॰ 9263 de 12 de janeiro de 1996. Regula o parágrafo 7 do artigo 226 da Constituição Federal, que trata do planejamento familiar. Brasília: Diário Oficial da União., 20 de agosto de 1997.p. 179.

10. Altman DG. Pratical statistics for medical research. London: Chapman; |99|.

Aceito para publicação: 13/1 |/2005 